\title{
Atomistic modeling of defect diffusion in SiGe
}

\author{
P. Castrillo, R. Pinacho, J. E. Rubio, L. M. Vega and M. Jaraiz \\ Department of Electronics, University of Valladolid, 47011 Valladolid, Spain \\ pedcas@tel.uva.es
}

\begin{abstract}
We have developed an atomistic model for dopant diffusion in SiGe structures and we have implemented it in the kinetic Monte Carlo process simulator DADOS. The model takes into account (i) composition and stress effects on the diffusivity of interstitials, vacancies and dopants, (ii) SiGe interdiffusion, (iii) dopant segregation and (iv) the modifications of band-gap and charge levels. The model has been tested for B and Sb providing a very good agreement with available experimental data.
\end{abstract}

\section{Introduction}

SiGe alloys are becoming increasingly important for the manufacture of current high performance devices. In consequence, it is crucial to be able to predict final profiles for dopants in SiGe deep-submicron devices taking into account the many factors that contribute to dopant diffusion. Among these, stress and composition increase the complexity already present in silicon processing modeling [1-3]. Namely, composition and built-in stress can alter formation and migration energies, band-gap and charge levels. As a consequence, they will influence dopant behavior through changes in the effective diffusivity and segregation. Moreover, composition and stress may vary spatially, due to inhomogeneities or to heterostructures, and with time, mainly due to Si-Ge interdiffusion. Given the complexity of such processing scenarios it is important to have a microscopic, physically-based model that can account for every component mechanism. Atomistic kinetic Monte Carlo (KMC) simulators are particularly appropriate for this purpose [4]. Using the KMC simulator DADOS we have developed a comprehensive model and a efficient simulation scheme that reveals the rich phenomenology associated to dopant diffusion in the SiGe system.

\section{Implementation and results}

As a non-lattice KMC simulator, DADOS follows only the atomistic defects, not the lattice ( $\mathrm{Si}$ or $\mathrm{Ge}$ ) atoms. The simulation domain has a 3D grid of small boxes (around $1 \mathrm{~nm}^{3}$ ) with varying composition and stress. The Ge content of the $\mathrm{Si}_{1-\mathrm{x}} \mathrm{Ge}_{\mathrm{x}}$ alloy is described by the discrete number of Ge atoms in each box. The composition dependence of the transport capacity of interstitials (I) and vacancies (V) in equilibrium conditions $\left(\mathrm{DC}_{\mathrm{I}}{ }^{*}\right.$ and $\mathrm{DC}_{\mathrm{V}}{ }^{*}$ ) has been modeled through changes in their formation energies and entropies ( $E_{\text {form }}$ and $s_{\text {form }}$ ), which have been assumed to scale linearly with Ge content. Figure 1.a illustrates the energy landscape that would see a diffusing particle (with migration energy $E_{m}$ ) when moving through a region where $E_{\text {form }}$ changes with the position. 

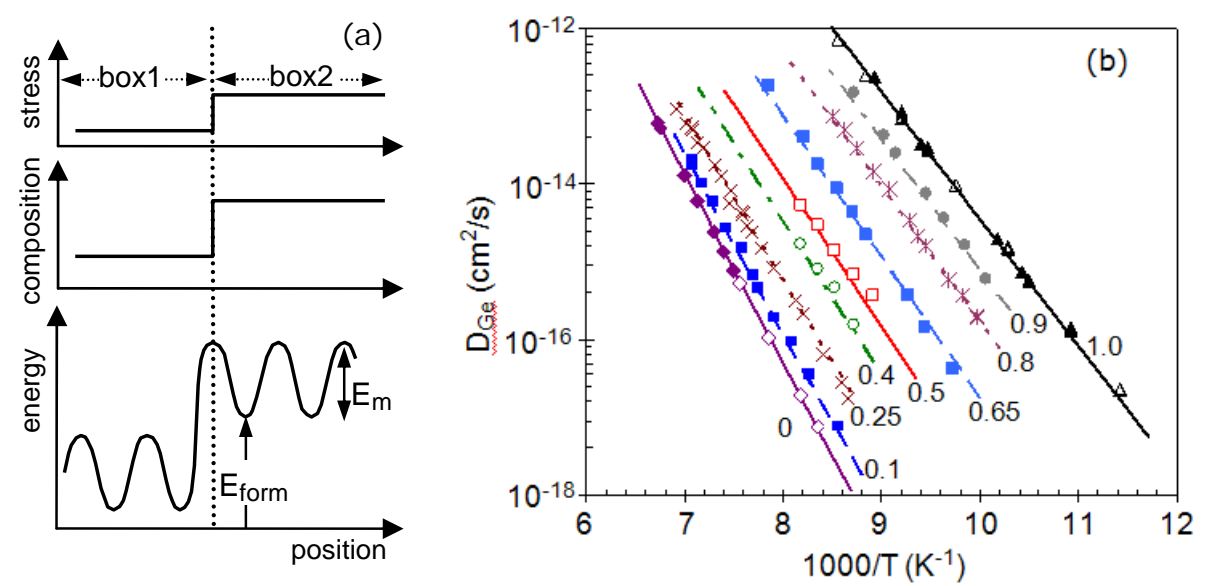

Figure 1: (a) Simplified energy scheme with position-dependent stress and composition. (b) Ge self-diffusivity in $\mathrm{Si}_{1-\mathrm{x}} \mathrm{Ge}_{\mathrm{x}}$ for different values of $\mathrm{x}$.

Symbols: experimental data, from Refs.[5-7]. Lines: present model.

We account for the different $\mathrm{Si}$ and Ge self-diffusivities by defining the relative probability of an I (or a V) moving a Ge or a Si atom. This relative probability is described by an activation energy and an entropy that, again, scale linearly with the Ge content. These simple assumptions lead to a very good fit of experimental data of $\mathrm{Ge}$ and $\mathrm{Si}$ diffusivities $\left(\mathrm{D}_{\mathrm{Ge}}\right.$ and $\left.\mathrm{D}_{\mathrm{Si}}\right)$ in the whole range of SiGe compositions [5-7]. As an example, $D_{G e}$ is shown in Figure 1.b. In our atomistic scheme, Si-Ge interdiffusion in SiGe heterostructures results from the probabilistic net exchange of Si and Ge atoms between neighbor boxes through the jump of individual Is or Vs form one box to another. The effects of I (or V) supersaturation on Si-Ge interdiffusion are automatically included.

Dopant diffusion is mediated by mobile dopant-defect pairs (such as BI and SbV). Thus, dopant diffusivity and segregation can be successfully fitted to experimental data [2, 8-11] by calibrating the $\mathrm{E}_{\text {form }}$ of dopants and the charge levels of dopantdefect pairs. The composition dependence of the SiGe band structure plays a role in dopant diffusion, specially in extrinsic conditions, and it has been carefully taken into account in the model.
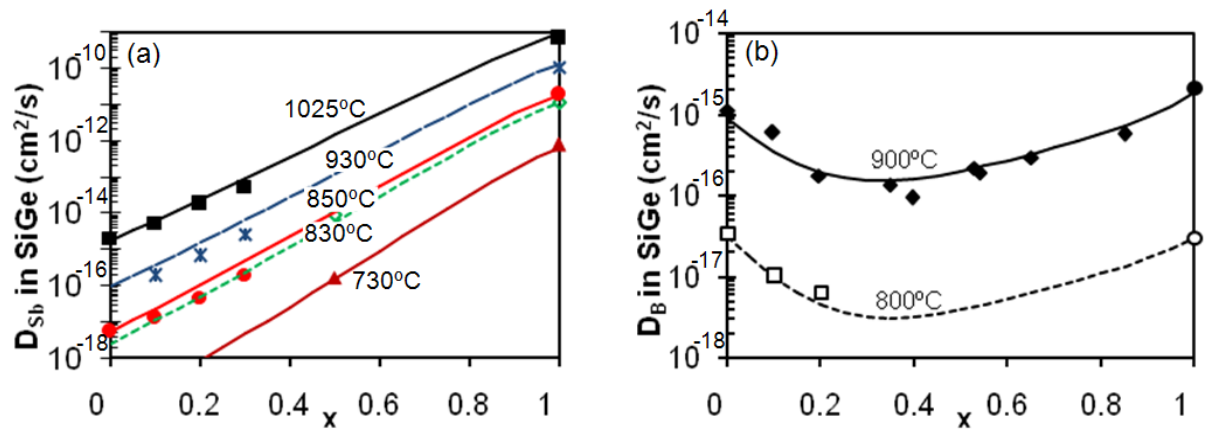

Figure 2: (a) Antimony and (b) boron diffusivities in $\mathrm{Si}_{1-\mathrm{x}} \mathrm{Ge}_{\mathrm{x}}$ as a function of $\mathrm{Ge}$ content. Symbols: experimental measurements [2, 8-10, 12]. Lines: model. 

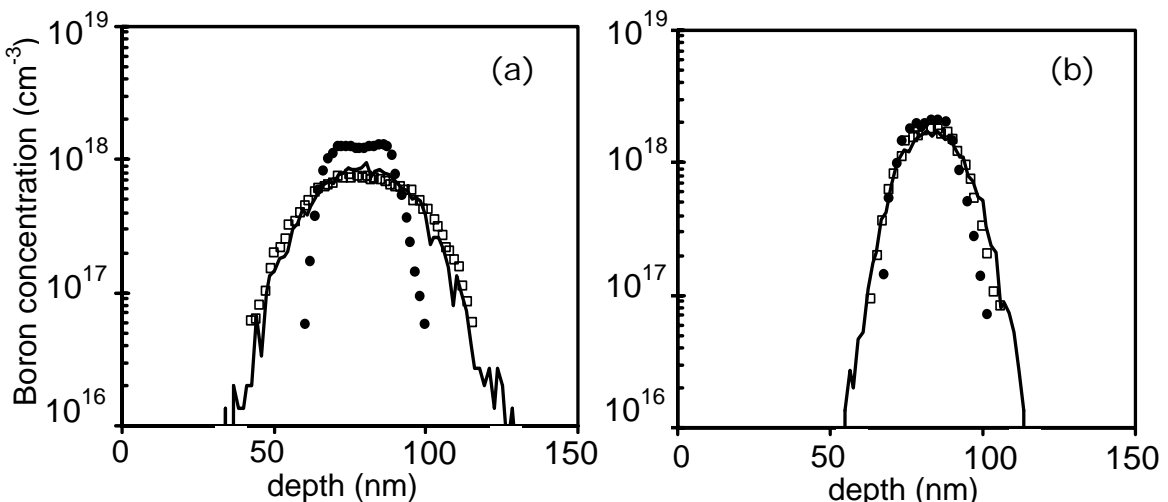

Figure 3: Diffusion of a B spike (a) in Si and (b) in strained $\mathrm{Si}_{0.83} \mathrm{Ge}_{0.17}$ after a 30 min., $860^{\circ} \mathrm{C}$ anneal. Dots: as grown. Squares: experimental data [13]. Lines: simulated

As an example, $\mathrm{D}_{\mathrm{Sb}}$ and $\mathrm{D}_{\mathrm{B}}$ as a function of composition are shown in Figures 2.a and 2.b. In our calibration, microscopic parameter values were kept within the ranges allowed by experimental or theoretical data available.

Stress has been implemented for a biaxial geometry. In this configuration, it is necessary to account for the experimental modification of the diffusivity in the perpendicular direction, characterized by an effective activation volume, and for the anisotropy, inferred from hydrostatic tests [1]. We model these features with an additional change of $\mathrm{E}_{\text {form }}$ and including the anisotropy in the migration rates of dopant-defect pairs.

Figures 3.a and 3.b illustrate the different B diffusion in Si and in SiGe (strained to $\mathrm{Si}$ ) [13]. The boron diffusion retardation in the depth direction is due to the joint effect of composition, biaxial compression and reduction of extrinsic diffusivity.

Boron segregation from $\mathrm{Si}$ to SiGe is well reproduced in the experiment shown in Figure 4.a [11]. We would like to point-out that: (i) in our model, B segregation is described by a decrease of boron formation energy in SiGe, not by the assumption of Ge-B pairs [11], and (ii) diffusivity and segregation can be calibrated independently.
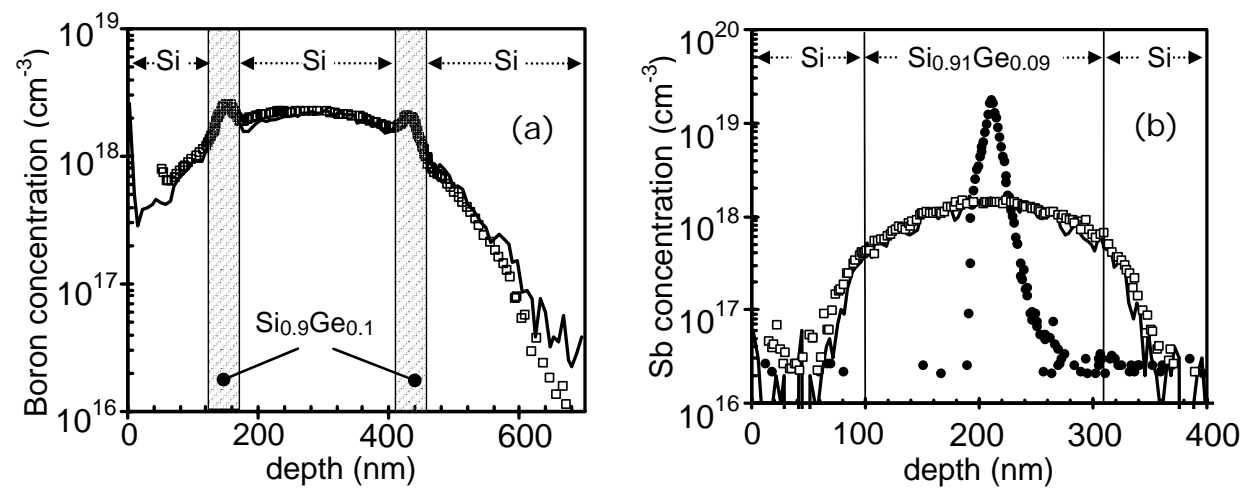

Figure 4: (a) Boron concentration profile, exhibiting segregation to SiGe layers after 96 hours at $850^{\circ} \mathrm{C}$. Squares: experimental data [11]. Lines: simulation.

(b) Diffusion of an Sb spike in a Si/SiGe/Si structure after $30 \mathrm{~min}$ at $1028^{\circ} \mathrm{C}$.

Dots: as grown. Squares: experimental data [2]. Lines: simulation. 
Finally, Figure 4.b shows the retardation of an antimony spike diffusion as it enters a $\mathrm{Si}$ region in a $\mathrm{Si} /$ strained $\mathrm{SiGe} / \mathrm{Si}$ structure [2].

In conclusion, an atomistic KMC implementation of microscopic, physically-based models can provide a wide-scope simulation environment for the complexities involved in current SiGe device processing. As an improvement over continuumbased implementations, KMC gives direct and easy access to every microscopic parameter and mechanism.

\section{Acknowledgements}

This work has been supported by the Spanish Government and the Castilla y Leon regional Government under projects TEC2005-05101 and VA070A05, respectively.

\section{References}

[1] M. Aziz, Y. Zhao, H. J. Gossmann, S. Mitha, S. P. Smith and D. Schiferl, "Pressure and stress effects on the diffusion of B and Sb in Si and Si-Ge alloys”, Physical Review B, vol. 73, pp. 054101/1-20, 2006.

[2] P. Kringhøj, A. Nylandsted-Larsen and S. Y. Shirayev, "Diffusion of Sb in strained and relaxed Si and SiGe”, Physical Review Letters, vol. 76, pp. 3372-3375, 1996.

[3] C. H. Chen , U. M. Gösele and T. Y. Tan, "Dopant diffusion and segregation in semiconductor heterostructures. Pt. 2. B in $\mathrm{Ge}_{\mathrm{x}} \mathrm{Si}_{1-\mathrm{x}} / \mathrm{Si}$ structures”, Applied Physics A, vol. 68, pp. 19-24, 1999.

[4] M. Jaraiz, P. Castrillo, R. Pinacho, I. Martin-Bragado and J. Barbolla, “Atomistic FrontEnd Process Modelling: A Powerful Tool for Deep-Submicron Device Fabrication”, Simulation of Semiconductor Processes and Devices 2001, Springer-Verlag, ISBN_3211-83708-6 10-17, 2001.

[5] N. R. Zangenberg, J. Lundsgaard-Hansen, J. Fage-Pedersen and A.Nylandsted-Larsen, "Ge self-diffusion in epitaxial $\mathrm{Si}_{1-\mathrm{x}} \mathrm{Ge}_{\mathrm{x}}$ layers", Physical Review Letters, vol. 87, pp.125901/1-4, 2001.

[6] A. Strohm, "Self-diffusion of ${ }^{71} \mathrm{Ge}$ in Si-Ge", Ph.D. Thesis, University of Stuttgart, 2002.

[7] M. Werner, H. Mehrer and H. D. Hochheimer, "Effect of hydrostatic pressure, temperature, and doping on self-diffusion in germanium”, Physical Review B, vol. 32, pp. 3930-3937, 1985.

[8] P. Laitinen, "Self- and impurity diffusion in intrinsic relaxed silicon-germanium”, Ph.D Thesis, University of Jyväskylä, 2004.

[9] P. Kuo, J. L. Hoyt, J. F. Gibbons, J. E. Turner and D. Lefforge, "Effects of strain on boron diffusion in Si and $\mathrm{Si}_{1-\mathrm{x}} \mathrm{Ge}_{\mathrm{x}}$ ”, Applied Physics Letters, vol. 66, pp. 580-582, 1995.

[10] S. Uppal, A. F. W. Willoughby, J. M. Bonar, N. E. B. Cowern, T. Grasby, R. J. H. Morris and M. G. Dowsett, "Diffusion of boron in germanium at $800-900^{\circ} \mathrm{C}$ ", Journal of Applied Physics, vol. 96, pp. 1376-1380, 2004.

[11] R. F. Lever, J. M. Bonar and A. F. Willoughby, "Boron diffusion across silicon-silicon germanium boundaries”, Journal of Applied Physics, vol. 83, pp. 1988-1994, 1998.

[12] Experimental data of Ref.9 have been corrected by a factor 0.3 in Fig.2.b in order to get commonly accepted values for B diffusivity in unstrained Si (about $4 \cdot 10^{-17} \mathrm{~cm}^{2} / \mathrm{s}$ ).

[13] P. Kuo, J. L. Hoyt, J. F. Gibbons, J. E. Turner, R. D. Jacowitz and T. I. Kamins, "Comparison of boron diffusion in $\mathrm{Si}$ and strained $\mathrm{Si}_{1-\mathrm{x}} \mathrm{Ge}_{\mathrm{x}}$ epitaxial layers", Applied Physics Letters, vol. 62, pp. 6126-14, 1993. 